LA-UR- $03-1739$

Approved for public release;

distribution is unlimited.

$\mathrm{C}$.
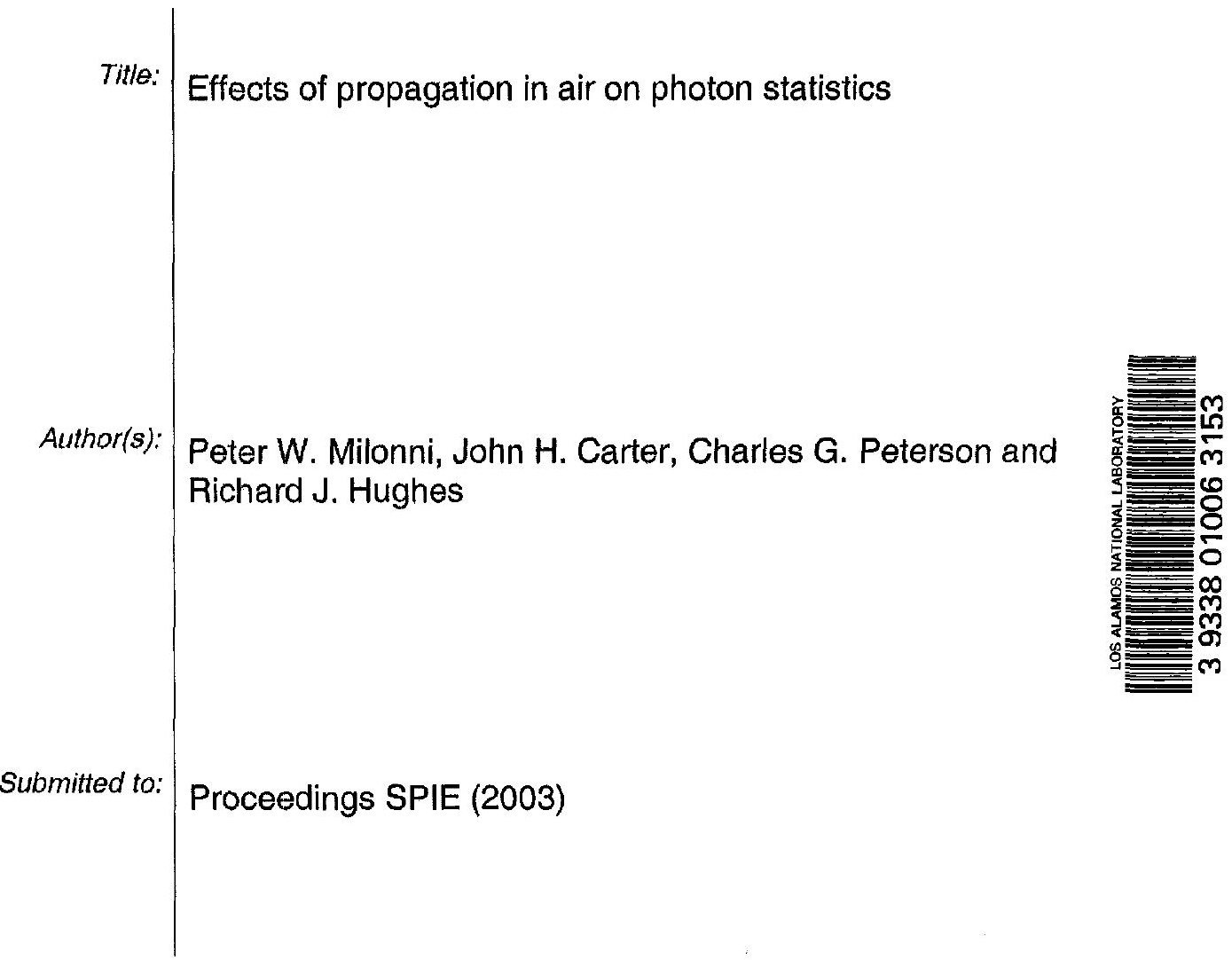

1

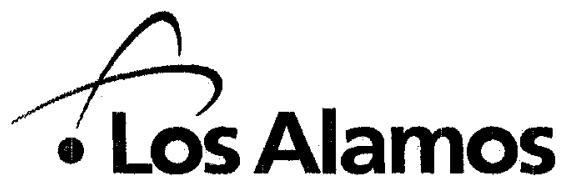

NATIONAL LABORATORY

Los Alamos National Laboratory, an affirmative action/equal opportunity employer, is operated by the University of California for the U.S. Department of Energy under contract W-7405-E.NG-36. By acceptance of this article, the $\equiv$ her recognizes that the U.S. Government retains a nonexclusive, royalty-free license to publish or reproduce the published form of t $D$ ntribution, or to allow others to do so, for U.S. Government purposes. Los Alamos National Laboratory requests that the publisher identify this article as work performed under the auspices of the U.S. Department of Energy. Los Alamos National Laboratory strongly supports academic freedom and a researcher's right to publish; as an institution, however, the Laboratory does not endorse the viewpoint of a publication or guarantee its technical correctness. 


\title{
Effects of propagation in air on photon statistics
}

\author{
Peter W. Milonni ${ }^{a}$, John H. Carter ${ }^{a}$, Charles G. Peterson ${ }^{b}$ and Richard J. Hughes ${ }^{b}$ \\ ${ }^{a}$ Theoretical Division, Los Alamos National Laboratory, Los Alamos, NM, USA 87545 \\ ${ }^{b}$ Physics Division, Los Alamos National Laboratory, Los Alamos, NM, USA 87545
}

\begin{abstract}
Calculations of photon counting distributions and fade probabilities are compared with measured data obtained using very weak laser pulses propagated over $10-\mathrm{km}$ horizontal paths. Good agreement is obtained between theory and experiment using various simplifying assumptions and values of the refractive-index structure constant $C_{n}^{2}$ lying within an expected range.
\end{abstract}

Keywords: Photon statistics, atmospheric turbulence, Kolmogorov theory

\section{INTRODUCTION}

It is of interest for various applications to know how the photon counting statistics of laser radiation are affected by propagation in the turbulent atmosphere. The theory of the effect of turbulence on the photon counting distribution, based on the general Mandel formula, ${ }^{1}$ is well established, but there appears to be insufficient experimental data to allow one to check the validity of different formulas that have been proposed for the modulation of the mean photon count. In this paper we compare calculations of photon counting distributions with measurements taken for horizontal propagation paths in the atmosphere.

Needless to say, the calculations must be based on certain assumptions about the nature of the atmospheric turbulence. The standard Kolmogorov model assumed here involves the refractive-index structure constant $C_{n}^{2}$, which cannot be reliably computed from first principles. Agreement between theory and experiment can be considered satisfactory if assumed values of $C_{n}^{2}$ within a range consistent with other kinds of experiments yield results for photon counting statistics that conform well to measured data. Conversely, it is possible to deduce, from the measured data and Mandel's formula, a distribution function for the modulation of the mean intensity produced by atmospheric turbulence.

In the following section we summarize some results from the theory of photon statistics and the theory of propagation of radiation in the turbulent atmosphere. Section 3 compares theoretical results for fade probabilities and counting distributions with measured data and outlines plans to extend this preliminary work.

\section{PHOTON ĆOUNTING AND TURBULENCE}

We will make the following simplifying assumptions. Background radiation noise is assumed to be negligible, and photoelectrons are counted by a detector whose area is small compared to the coherence area of the incident field. The photoelectron counting interval $T$ is taken to be small compared to both the coherence time $\tau_{c}$ of the laser source and the characteristic time scale $\tau_{a}$ of the atmospheric fluctuations. As a further simplifying assumption we will also assume that the incident field from the laser is well approximated by a plane wave. A more general analysis going beyond these approximations and addressing a wider range of experimental data is in progress and will be presented elsewhere.

The probability $p(n, T)$ of counting $n$ photons in a time interval $T$ is given by ${ }^{1}$

$$
p(n, T)=\left\langle\frac{\xi^{n}}{n !} X^{n}(t, T) e^{-\xi X(t, T)}\right\rangle
$$

where $\xi$ characterizes the detection efficiency and

$$
X(t, T)=\int_{t}^{t+T} d t^{\prime} I\left(t^{\prime}\right)
$$

$I\left(t^{\prime}\right)$ is the radiation intensity at time $t^{\prime}$, and $t$ is the time at which the counting measurement begins. The average in equation (1) is over these starting times $t$. In the experiments of interest the radiation is in the form 
of $\sim 1 \mathrm{~ns}$ laser pulses with a repetition rate of $1 \mathrm{MHz}$, so that there are $10^{4}$ pulses during the counting interval $T=10 \mathrm{~ms}$. Then $X(t, T)$ may be taken to be independent of the starting times of successive counting intervals. In this approximation

$$
p(n, T)=\frac{q^{n}}{n !} e^{-q}
$$

where $q=\xi \int_{t}^{t+T} d t^{\prime} I\left(t^{\prime}\right)$ is the mean number of photons counted in an interval $T$.

The measured photon statistics depend on the modulation of the mean photocount caused by the propagation of the radiation through the turbulent atmosphere. Denoting the probability distribution function for this random modulation by $P(q)$, we replace (3) by

$$
p(n)=\int_{0}^{\infty} d q \frac{q^{n}}{n !} e^{-q} P(q)
$$

where the dependence on $T$ is now implicit.

It is well known that the Kolmogorov theory of turbulence and dimensional analysis imply a refractive-index structure function $D_{n}(r)=\left\langle[n(r)-n(0)]^{2}\right\rangle=C_{n}^{2} r^{2 / 3}$ for isotropic turbulence and $r$ much greater than the inner scale of turbulence and much smaller than the outer scale. The structure constant $C_{n}^{2}$ varies between roughly $10^{-17} \mathrm{~m}^{-2 / 3}$ under good atmospheric seeing conditions and $10^{-13} \mathrm{~m}^{-2 / 3}$ under poor seeing conditions.

There is a large literature, based on the Kolmogorov theory, on the propagation of light in the turbulent atmosphere. ${ }^{2}$ For the purpose of using the Mandel formula (4) we are interested specifically in the probability distribution $P(q)$ associated with intensity scintillations. (See Dravins et al. ${ }^{3}$ and references therein.) The most frequently assumed distribution for intensity scintillations is based on the assumption that the intensity modulations associated with successive path intervals are multiplicative and independent, so that the logarithm of the intensity is the sum of many independent perturbations. The central-limit theorem then implies a normal distribution for the logarithm, or a $\log$-normal distribution for $P(q)$ :

$$
P(q)=\frac{1}{\sigma q \sqrt{2 \pi}} e^{-\left[\log (q / \bar{q})+\frac{2}{2} \sigma^{2}\right]^{2} / 2 \sigma^{2}}
$$

where $\bar{q}$ is the mean measured count in the interval $T . \sigma$ is the variance of $\log q$, and is related to the normalized variance in $q\left(\sigma_{q}^{2}=\left\langle(q-\bar{q})^{2}\right\rangle / \bar{q}^{2}\right)$ by $\exp \left(\sigma^{2}\right)-1=\sigma_{q}^{2}$.

The Rytov approximation ${ }^{2}$ leads to the following expression for plane-wave propagation:

$$
\sigma^{2}=1.23 C_{n}^{2} k^{7 / 6} L^{11 / 6}
$$

where $k=2 \pi / \lambda, \lambda$ is the wavelength, and $L$ is the propagation distance. The results (5) and (6) are valid in the weak-perturbation limit of small $\sigma$. Experiments by Gracheva and Gurvich ${ }^{4}$ indicated that the log-normal distribution is reasonably accurate for $\sigma^{2}<0.3 .^{5}$ Using (6) with $\lambda=772 \mathrm{~nm}$ and $L=10 \mathrm{~km}$, their results would imply that the log-normal distribution should be accurate for $C_{n}^{2}<9.8 \times 10^{-17} \mathrm{~m}^{-2 / 3}$. It must be noted, however, that (6) is based on the plane-wave assumption; for spherical waves, for instance, equation (6) must be multiplied by 0.4 , which results in $C_{n}^{2}<2.5 \times 10^{-16} \mathrm{~m}^{-2 / 3}$ for the validity of the log-normal approximation, assuming that the condition of validity is $\sigma^{2}<0.3$.

Theoretical studies of the effects on photon statistics of propagation through the turbulent atmosphere were discussed many years ago by Diament and Teich, ${ }^{6}$ among others. In spite of many years of research into the propagation of electromagnetic waves in the turbulent atmosphere, there appear to be few experimental studies of the effects of atmospheric propagation on photon statistics. The most extensive studies appear to be those of Dravins et $a l^{3}$, who noted that, "Although [the prediction of the log-normal distribution] is widely accepted as correct, there seem to exist but few experimental verifications to any substantial accuracy." Their Figure 2 and related discussion indicates that the scintillation of starlight is very well described by a log-normal distribution for values of $\sigma_{q}^{2}$ in the range of their measurements $\left(\sigma_{q}^{2}<0.4\right)$. This is consistent with the conclusions of Gracheva and Gurvich. ${ }^{4}$ Abdullaev et al. ${ }^{7}$ have measured photocounting distributions of laser radiation propagated over an inclined path of length $1 \mathrm{~km}$ and report good agreement with the log-normal theory for sawtooth and rectangular modulations of the laser intensity.

The experiments of interest here differ from previous ones in that they involve very weak (photon number $<1)$ repetitive short pulses, as described above, and long horizontal propagation paths. The photon counting data were taken as part of a more general experimental investigation of quantum key distribution. ${ }^{8}$ 


\section{COMPARISON OF THEORY AND EXPERIMENT}

We consider here two sets of data, the results for which are representative of about a dozen sets of data taken at different times. As a basic initial test it was verified that the counting distribution at the transmitter was Poissonian, as assumed above.

The fade probability, i.e., the probability of a count below the mean $\bar{q}$, is calculated using equation (4). (The surge probability $\left.P_{\text {surge }}=1-P_{\text {fade }}.\right)$ Figure 1 shows measured fade fractions obtained for a $10 \mathrm{~km}$ path in daylight, together with predicted fade probabilities based on equations (4) and (5) for three different values of $\sigma$. Assuming $\sigma=0.3$ based on the fade data, we plot $p(n)$ [equations (4) and (5)] in Figure 2 for the measured mean $\bar{q}=52.5$. Corresponding experimental data for $p(n)$ are shown in Figure 3. The variance $\left\langle(q-\bar{q})^{2}\right\rangle$ for the theoretical curve shown in Figure 2 is 306, compared to the measured variance of 296.

Figures 4 and 5 show corresponding results for a 10-km path at night. In this case we plot $p(n)$ (Figure 5 ) for $\bar{q}=20.1$ and $\sigma=0.61$. The predicted variance of the photon number is 202 , compared to the measured variance of 207.

As these results indicate, theoretical results based on the log-normal distribution are in good qualitative agreement with the measured data for values of $C_{n}^{2}$ within the well-established range. We have ignored in this preliminary investigation the effects of background and detector noise, which are relatively small in the regime of operation. ${ }^{8}$

The assumption of log-normal statistics for the random intensity modulations associated with atmospheric turbulence has been validated for stellar scintillations by the work of Dravins et al ${ }^{8}$ Strong evidence for the log-normal distribution for laser radiation propagated over horizontal paths is provided by some experimental studies, ${ }^{4,7}$ while others ${ }^{9,10}$ lend support to other types of distribution. The use of larger laser signals in the present experimental system ${ }^{8}$ should allow us to invert the Poisson transform relation (4) and determine the probability density $P(q)$ from measured counting distributions $p(n)$. In particular, by varying the path length it should be possible to test the scaling of the photocount variance predicted by equation (6), for instance, and to study the onset of saturation of the scintillations.

\section{REFERENCES}

1. L. Mandel, "Fluctuations of photon beams and their correlations," Proc. Roy. Soc. (London), 72, pp. $1037-1048,1958$.

2. V. I. Tatarski, Wave Propagation in a Turbulent Medium, McGraw-Hill, New York, 1961.

3. D. Dravins, L. Lindegren, E. Mezey and A. T. Young, "Atmospheric intensity scintillation of stars. I. Statistical distributions and temporal properties," Pub. Astron. Soc. Pac. 109, pp. 173-207, 1997.

4. M. E. Gracheva and A. S. Gurvich, "On strong fluctuations of the intensity of light when propagating in the lower layer of the atmosphere," Izv. Vuz. Radiofiz. 8, pp. 717-724, 1965.

5. R. L. Fante, "Electromagnetic beam propagation in turbulent media," Proc. IEEE 63, pp. 1669-1692, 1975. 6. P. Diament and M. C. Teich, "Photodetection of low-level radiation through the turbulent atmosphere," J. Opt. Soc. Am. 60, pp. 1489-1494, 1970.

7. S. S. Abdullaev, A. T. Mirzaev, and I. K. Rasulov, "Statistics of photocounts of modulated radiation transmitted by a turbulent atmosphere," Sov. J. Quantum Electron. 17, pp. 325-328, 1987.

8. R. J. Hughes, J. E. Nordholt, D. Derkacs and C. G. Peterson, "Practical free-space quantum key distribution over $10 \mathrm{~km}$ in daylight and at night," New J. Phys. 4, pp. 43.1-43.14, 2002.

9. G. Parry, "Measurements of atmospheric turbulence induced intensity fluctuations in a laser beam," Opt. Acta 28, pp. 715-728, 1981 .

10. J. H. Churnside and R. J. Hill, "Probability density of irradiance scintillations for strong path-integrated refractive turbulence," J. Opt. Soc. Am. 4, pp. 727-733, 1987. 


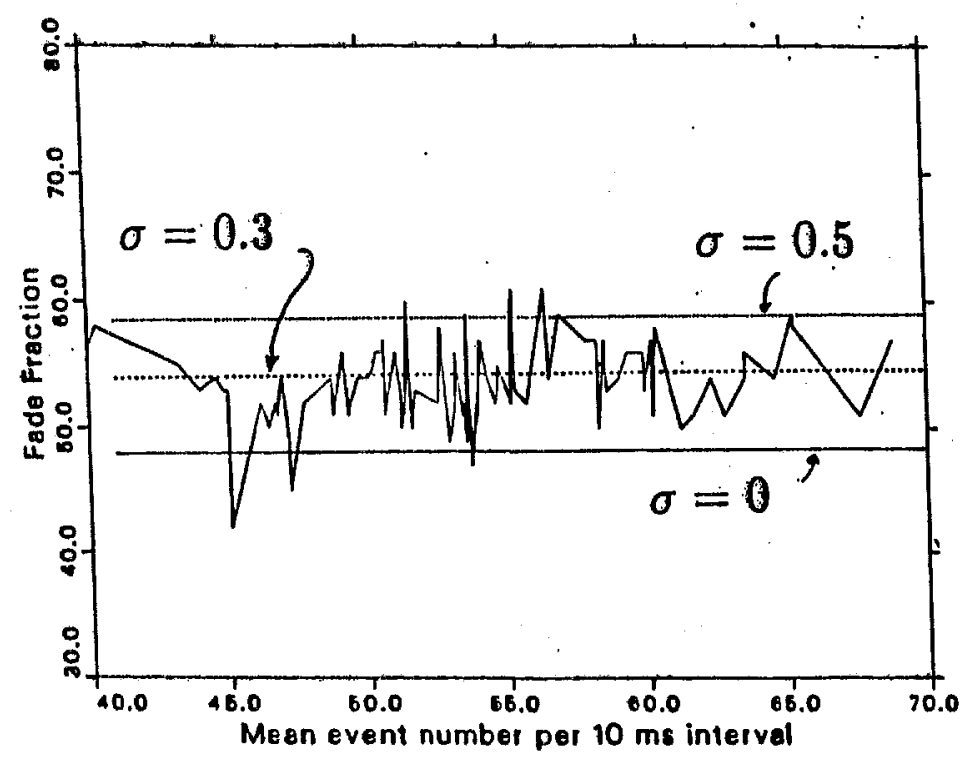

Figure 1. Measured fade fractions for a 10-km horizontal path in daylight, compared with computed fade probabilities for three values of $\sigma$.

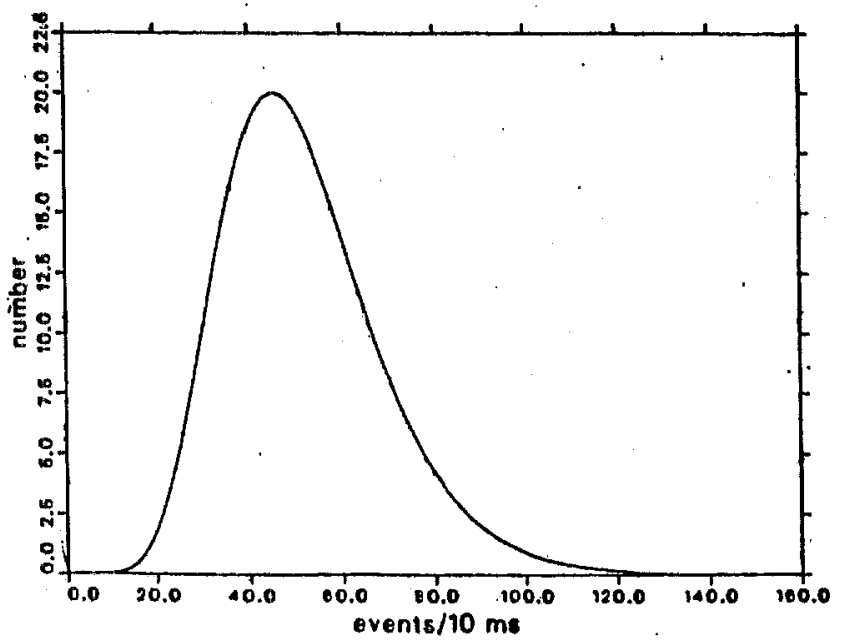

Figure 2. Computed counting distribution for $\overline{\bar{q}}=52.5$ and $\sigma=0.3$

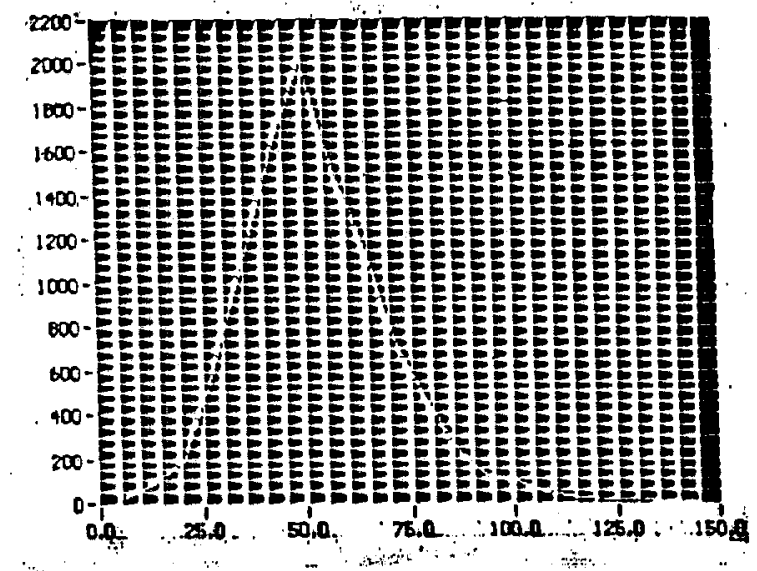

Figure 3, Measured photocount distribution in daylight. The mean and variance of thephotocounts are 52.5 and 296.3 , respectively. 


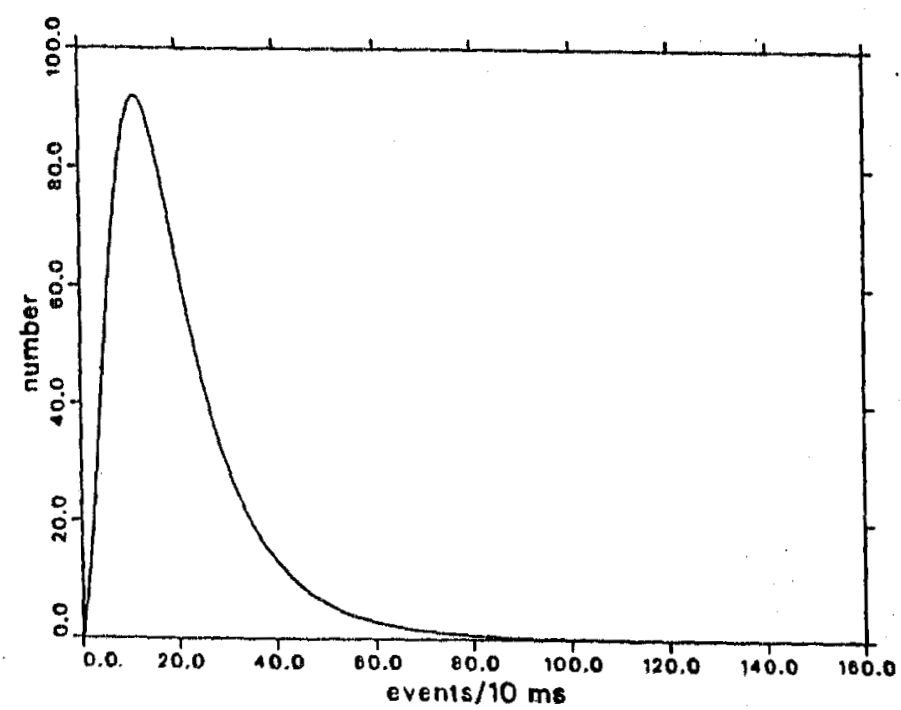

Figure 4. Computed counting distribution for $\bar{q}=20.07$ and $\sigma=0.01$

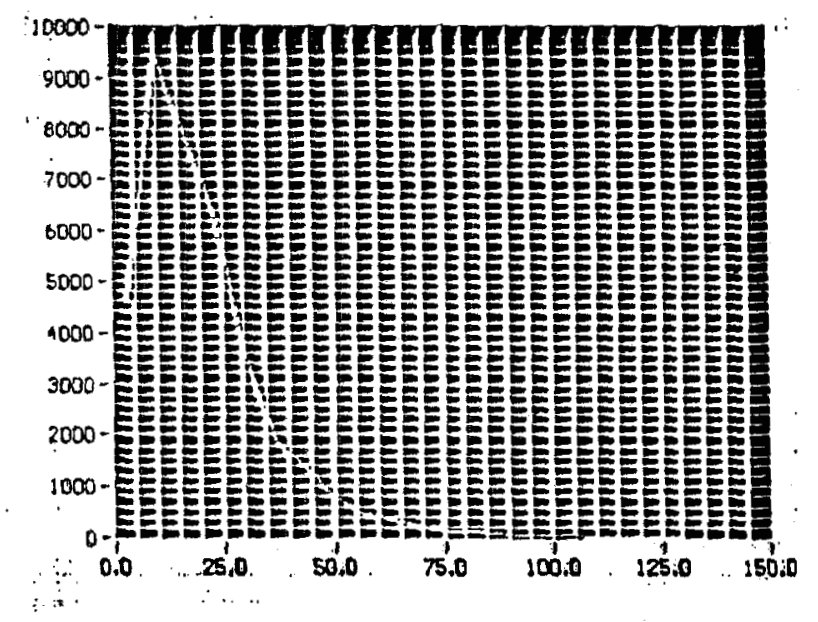

Figure 5. Measured photocount distribution at night. The mean and variance of thephotocounts are 20.1 and 207, respectively. 